\title{
An Integrated Platform Supporting Intangible Cultural Heritage Learning and Transmission: Definition of Requirements and Evaluation Criteria
}

\author{
Francesca M. Dagnino ${ }^{1}$, Leontios J. Hadjileontiadis ${ }^{2}$, Michela Ott ${ }^{1}$ \\ and Francesca Pozzi ${ }^{1}$ \\ ${ }^{1}$ Istituto Tecnologie Didattiche - CNR, Italy \\ ${ }^{2}$ Aristotle University of Thessaloniki, Greece
}

\begin{abstract}
The paper offers an experience-based viewpoint on two key phases of the development of an Information and Communication Technologies (ICTs)-based system: the definition of requirements and identification of related criteria and methodology for its evaluation. In doing so, it refers to the unique context of the i-Treasures EU project, which deals with the development of an innovative integrated platform to support the learning and transmission of Intangible Cultural Heritage (ICH). The i-Treasures integrated platform is conceived to support both traditional learning approaches and innovative and active learning processes, based on extensive use of sensor-technologies. In this light, during the development process, particular attention has been devoted to the definition of requirements with specific reference to sensor-mediated Human Computer Interaction (HCI) issues and the evaluation process was designed accordingly, in coherence with the specific advanced features of the integrated platform. The paper offers a view of the complexity of the design of ICT-based tools supporting the preservation and transmission of ICH and also provides an insight (and this could have a broader impact) into the methodology adopted to harmonize the requirements and the evaluation phases which are key pillars for the construction of any educationally effective ICT-based learning system.
\end{abstract}

Keywords: information systems, cultural heritage education, intangible cultural heritage, requirements, evaluation criteria, fuzzy logic, learning management systems

\section{Introduction}

The definition of requirements and the establishment of criteria for evaluation are two key aspects of the development of ICT-based learning systems. This is true, irrespective of the software development model adopted, ranging from more traditional (waterfall) models to recent, more adaptive approaches (Huo et al, 2004).

In the former case, the requirements collection/identification is the first step of the process and they are gathered before affording any subsequent design, development and verification and evaluation activity. In the latter case, a more dynamic, iterative and incremental approach to software development (Cockburn, 2008; Larman \& Basili, 2003) is adopted. Here, the phases of system design and requirements definition pave the way to the implementation and evaluation phases and the results of such evaluation inform back the original requirements, in a sort of cycle, which may take place even several times (Figure 1).

These concepts of iteration and incremental development have been taken up and integrated into the "agile" methodology (Kent et al., 2001), which is also based on the idea of a stronger and more continuous involvement of users in the software development process.

In the following, we describe the intertwined and recursive approach adopted in the i-Treasures project (Dimitropoulos et al., 2014) to define the requirements and establish the evaluation criteria.

This project, as it is explained below, is situated in the area of Intangible Cultural Heritage 

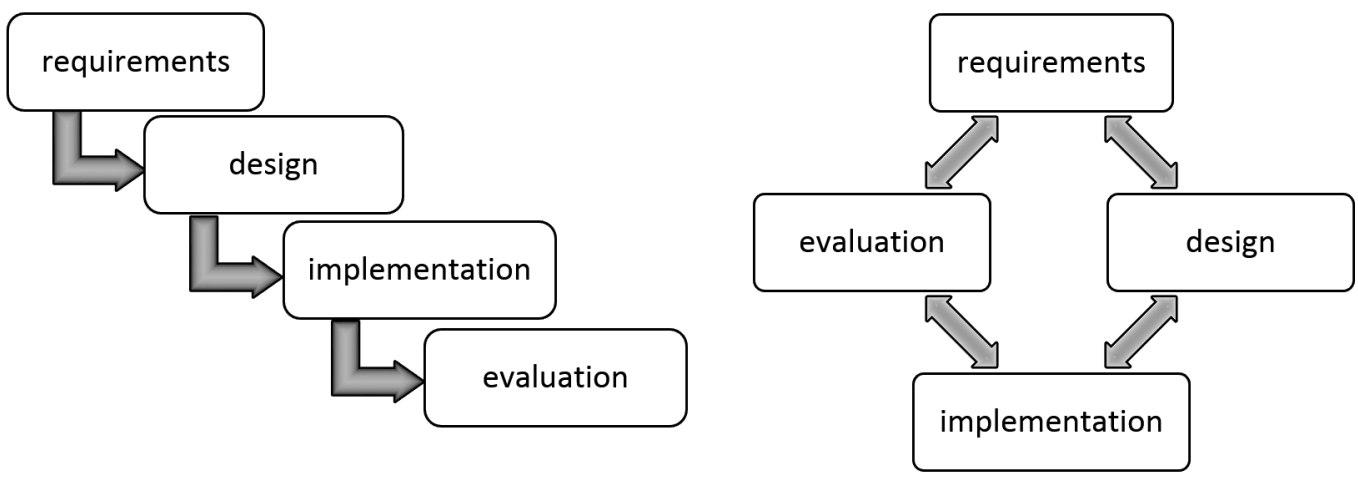

Figure 1. Waterfall/standard vs iterative and incremental approaches to software development.

(ICH) Education and is aimed "to develop an open and extendable platform to provide access to ICH resources, enable knowledge exchange between researchers and contribute to the transmission of rare know-how from Living Human Treasures to apprentices".

It encompasses a wide range of field experiments (yet to be implemented), which will point out also the perceived quality /usability /usefulness of the whole learning platform, with a particular focus on the implemented learning actions from the viewpoint of end-users.

The conceptual work done to develop the iTreasures platform well instantiates the strong links and interdependencies between the initial phases of software development (i.e.: requirements collection and overall design) and the subsequent ones (formative and summative evaluation).

Thus, in what follows, we first set the scene by casting a glance to the i-Treasures project and to the different types of tools typically supporting cultural heritage education. Subsequently, we provide further insight into the process of requirements identification and to the definition of related criteria for the evaluation of the developed software system, with a particular focus on Human Computer Interaction (HCI) aspects.

\section{Setting the Scene}

For better comprehension of the constraints and issues informing the development of the i-Treasures integrated platform, a general vision of the objectives of the i-Treasure project is presented in the following section, along with a brief overview of the tools that are adopted to sustain cultural heritage education.

\subsection{The i-Treasures Project}

i-Treasures is an Integrated Project co-financed by EU under the ICT theme (Information and Communication Technologies) of the FP7 ( $7^{\text {th }}$ Framework Program) which started in 2013 and will conclude its activities in 2017. It deals with ICH (Intangible Cultural Heritage) which, according to UNESCO (2003), encompasses all those "practices, representations, expressions, as well as the knowledge and skills (including instruments, objects, artefacts, cultural spaces), that communities, groups and, in some cases, individuals recognize as part of their cultural heritage".

i-Treasures considers a number of different ICHs, belonging to four different "use cases" (corresponding to four areas: singing, dancing, music and craftsmanship). Each use case is further instantiated in different "sub-use cases", namely:

- singing: Canto a Tenore, Canto in Paghjella, Byzantine music and Human BeatBox;

- dancing: Tsamiko, Căluş, Walloon and contemporary dances;

- craftsmanship: the art of pottery in Greece and France;

- music: contemporary music composition.

The ultimate aim of i-Treasures is to go far beyond the simple ICH dissemination and the mere digitization of cultural contents; rather, it aims at analyzing and modeling the different ICHs (in the fields of dancing, singing, music 
composition and craftsmanship), thus, supporting learning of the rare know-how behind these cultural expressions and their passing down to new generations.

As a matter of fact, the whole i-Treasures system has been conceived having in mind an educational objective and thus it is oriented to support a variety of educational and demonstration activities, ranging from simple historical information to practical/physical exercises and gaming challenges. It integrates, together with a powerful and adaptive Learning Management System (LMS), a number of different technological tools whose adoption is required by the peculiarity of the ICH field and of the project mission.

The envisaged learning process, which has been conceived as highly customizable in all its parts/ aspects, is expected to take learners beyond the concept of "imitation", by allowing them to:

- acquire relevant conceptual information in different formats (e.g., audio, video, narrative),

- view in details the performances of expert artists,

- acquire awareness in relation to the different elements at play during a performance (e.g., what happens in the vocal tract when a sound is produced)

- practice the ICH and receive appropriate feedback (in different formats, e.g., audio or video), so as to be able to adjust their performance and reach increased levels of competence in an easier way.

\section{2. i-Treasures and the Panorama of Cul- tural Heritage Education}

As said above, the i-Treasures system allows access to a number of specific tools, some of which specifically devoted to education. One of its main characteristic is the adoption of cutting edge technologies oriented to sustain high level of interaction and personalized learning paths allowing to meet a variety of specific users' learning needs. These characteristics frame the system in a very specific way in respect to other existing tools for Cultural Heritage Education.
As a matter of fact, according to Ott and colleagues (2014), it is possible to identify different typologies of digital tools for Cultural Heritage Education, that can be roughly categorized in:

1. Virtual Museums (VMs), i.e., ICT-based environments supporting virtual visits (via Internet or on-site through standalone applications) of past and present cultural heritage artifacts and expressions (Scrovegni Chapel; Giza 3D). Figure 2 shows the screenshot from an interesting Virtual Museum related to a patrician villa in ancient Roma (Livia's Villa), where users are allowed to explore the archaeological site, walk around and see the reconstructed building.

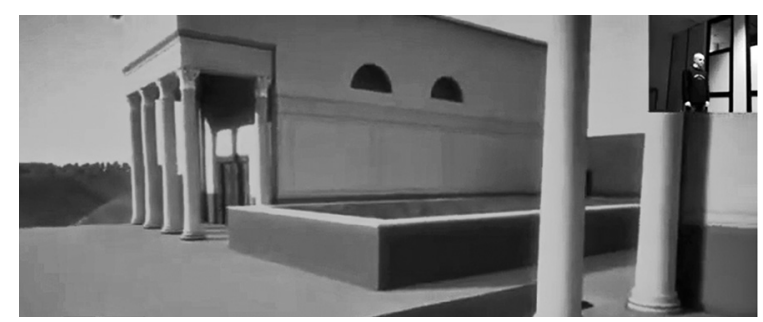

Figure 2. View from the Livia’s Villa Virtual Museum.

Some more sophisticated and educationally oriented VMs (Antonaci et al., 2013) also allow conducting immersive explorations (Etruscanning) by offering the possibility to carry out personalized paths and customize the view of $\mathrm{CH}$ objects (Figure 3 ).

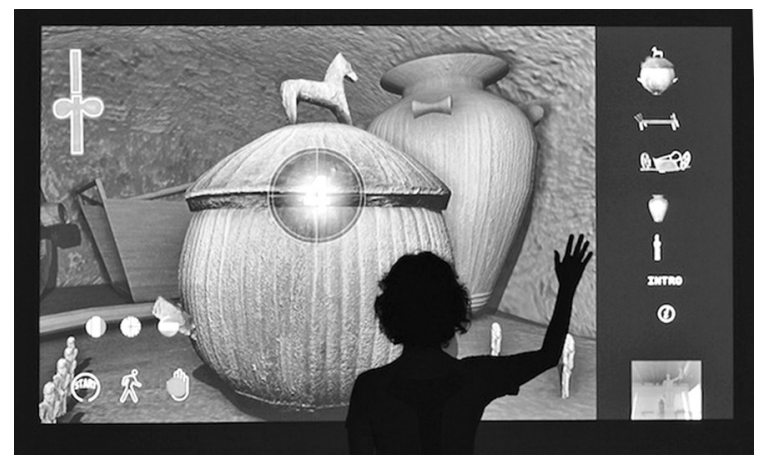

Figure 3. Etruscanning: advanced interaction.

2. Serious Games, i.e., gaming environments aimed at supporting learning in the field of cultural heritage. Such tools, by relying on basic game mechanics, offer educational experiences where the interaction between humans and the technology is wider and more advanced. Figure 4 shows a screenshot from 


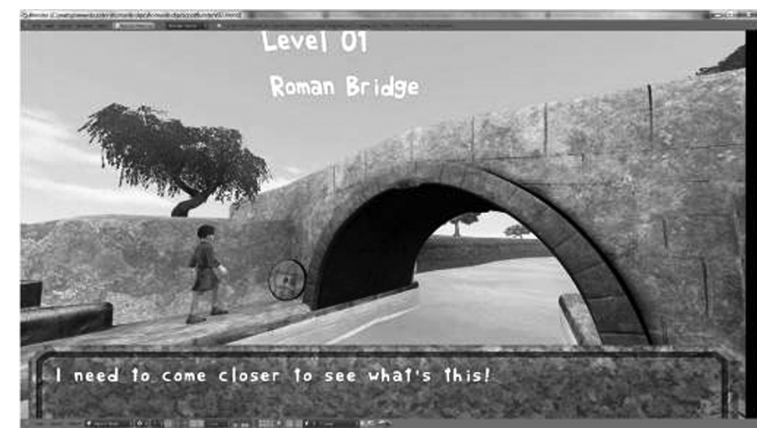

Figure 4. Screenshot from the APA game.

a Serious Game set in the $13^{\text {th }}$ century, oriented to inform about the life in the Roman Bologna (APA game).

3. ICT-based systems aimed at supporting active learning and practicing of cultural expressions, so as to allow the acquisition of competences and abilities (as opposed to other systems, more oriented to information delivery). An example is the system developed in the framework of the i-maestro project (www.i-maestro.org), which employs self-learning environments, gestural interfaces and augmented instruments, promoting new methods for music training.

These three different types of ICT tools supporting Cultural Heritage Education also instantiate different types and/or levels of interaction between technology and user (Ott \& Pozzi, 2008). Virtual Museums (VMs) use advanced 3D and virtual reconstruction technologies to allow an in-depth view of cultural heritage artifacts, but they often offer limited learning possibilities, mainly oriented to having an exhaustive view of the objects themselves, (e.g., choosing the item to focus on, zooming in and out, watching them from multiple perspectives). On the other hand, most Serious Games in the domain of Cultural Heritage offer advanced interaction from the pedagogical viewpoint, such as the possibility of customizing the learning paths and keeping track of the learners' behavior and suc- cesses/failures and are, therefore, more adaptive to meet specific users' learning needs.

The third category of ICT tools refers to the systems that can be quite different from one another, but share the characteristic of encompassing a variety of tools and sub-systems (so that they can be regarded and defined as integrated platforms), allowing a high level of interaction between humans and computer.

The i-Treasures system can be ascribed to this last category in that it:

- integrates and makes use of a variety of different tools supporting the adoption of cutting-edge ICT-enhanced technologies, which per se represent a true innovation in the field of Cultural Heritage education (Gaitatzes et al., 2001; Veltman, 2005; Branchiesi, 2006)

- relies on structured and complex interaction modalities in order to allow the reproduction of dynamic events. In fact, besides providing information and sustaining theoretical knowledge, the learning/teaching interventions foreseen in i-Treasures also have the aim of passing down practical competences and abilities instantiated in precise actions/movements/gestures/sounds (Figure 5).

\section{The i-Treasures System: Key Features}

The i-Treasures integrated platform has the primary aim to enable the widest possible participation of communities, groups and individuals in the safeguarding and transmission of ICH. It gives access to different types of content (e.g. text, audio, images, video, 3D graphics) and offers a wide range of tools supporting varied educational experiences.

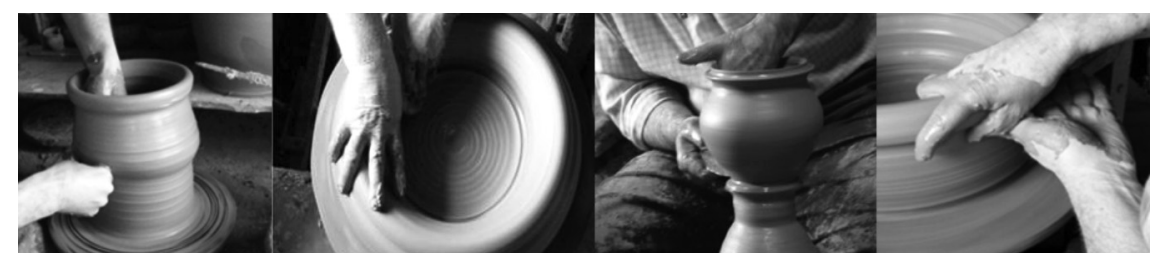

Figure 5. i-Treasures: passing down the specific hand movements for pottery making. 


\subsection{Overall Structure}

The i-Treasures system is conceptually subdivided into four modules, namely:

- Module A - ICH capture and analysis.

- Module B - Data fusion and semantic analysis.

- Module C-3D visualization module for sensorimotor learning.

- Module D - Web platform for research and education.

These constitute the backbone of the system architecture, as illustrated in Figure 6.

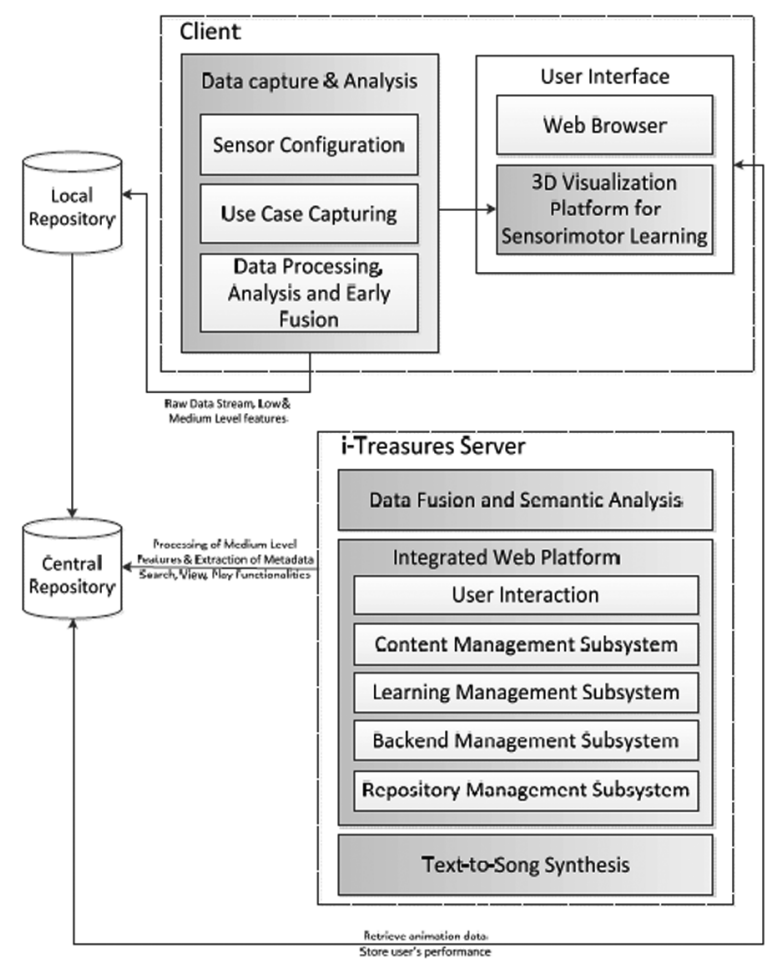

Figure 6. The architecture of the i-Treasure system.

In particular:

A. As to the multimodal sensing technology,first of all, relevant and detailed information on different artistic expressions is gathered from expert performers and researchers in the field; then, based on this information and thanks to Module A, key features of the ICHs are "captured" by using advanced ICT- and sensorbased technologies during experts' performances.

For example:
- as to singing, specific vocal emissions are captured, by tracking the vocal tract of the singers during performance;

- as to dance, body motion, posture and movements are captured, coded and registered by means of advanced motion capture techniques;

- as to pottery, fingers and hand gestures are tracked and coded;

- as to music composition, affective aspects are revealed and coded by adopting advanced neurophysiological tracking and measuring methods and tools.

B. A thorough process of modeling data (Analysis \& Modeling- Multimodal data fusion \& Semantic Media interpretation) is then carried out by relying on advanced Semantic Multimedia Analysis techniques (Lakka et al., 2011). In particular, image/signal processing and pattern recognition techniques are applied to recorded signals to extract low (e.g. motion tracking) and medium (media patterns, e.g. postures, actions, audio tempos etc.) level features. Data fusion analysis is applied to exploit information across different modalities, while context and content are integrated for mapping the set of low or medium-level multimedia features to highlevel concepts using probabilistic inference, i.e. transforming the extracted data into a level of interpretation understandable by humans.

The two above mentioned processes lead to and allow shaping and directing the learning process of apprentices. In fact, the system foresees that learners are exposed to multi-modal and multisensory learning experiences, carrying out individual trials and receiving appropriate feedback, so as to reach increased levels of competence in an easier, more direct, quicker and effective way (Dias et al., 2014; Pozzi et al., 2014). In this line:

C. Taking advantage of recent advances in webbased game engines 3D technology (3D platform) is used to develop a learning environment which enhances training and evaluation of the learner's performance by means of sensorimotor learning. Using multimodal inputs from different sensors, the application enables users to create an avatar, i.e. a $3 \mathrm{D}$ representation of them that visualizes 
their motions. An AI based virtual tutor corrects/ manipulates/guides the user to help in mastering the ICH. The application compares user inputs with the master inputs in the database and evaluates the performance of the user.

D. A specific web platform is dedicated to learning and educational activities. It is conceived as an open-source content management system offering a variety of features with significant added value: automated templates, multi-lingual and universal access to the content, scalable expansion, easily editable content, access and version control, scalable feature sets with installable software plug-ins or modules to extend functionality, etc. As shown in Figure 6, it consists of five different sub-systems supporting: 1) user interaction (browsing, searching, viewing, playing), 2) content management 3) learning management 4) back-end management of the web platform (user management, template management, etc.), and 5) database management. The main focus of interest on the central database of this educational platform are the metadata repository and the corresponding knowledge base (instantiated ontologies) that host the products of the semantic analysis. The overall educational aspects of the platform are technically realized by a seamlessly interoperable Learning Management System (LMS) for planning, implementing and assessing learning processes which support different educational scenarios, by adopting a User Centered Design (UCD) approach (Bødker, 2000; Abras et al., 2004). In these scenarios, users/learners are supposed to interact with the platform in order to:

- acquire information about the specific ICH (e.g., understanding cultural background, viewing/listening to performances)

- practise the specific ICH and be enabled to act as performers (thus gaining practical competences).

As already mentioned, while the former objective could be easily pursued using traditional learning methodologies (e.g., including individual study of resources, infield data collection, discussion with the experts, quizzes to test acquired theoretical knowledge), reaching the latter objective is strongly dependent on the combined use of cutting edge ICT and sensor technologies.

\subsection{Sensors' Role and Mediation}

From a general viewpoint, the i-Treasures system instantiates a distinctive situation, where the innovative, diffused use of multiple sensors plays a major role and affects relevant aspects of the human-computer interaction.

Actually, two different types of sensor-based learning processes take place in the different directional phases of the project:

- one that "goes" from humans to computer (originated by sensors capturing the performers' movements, which are then modeled; in this case the computer learns from humans) and

- one that "goes" from computer to humans (humans learn from the computer and sensors help the computer in interpreting and assessing human performances).

In Figure 7, Line A represents the sensor-mediated path from humans to computer and viceversa, while Line B represents the more traditional interactions between the computer and humans and vice-versa.

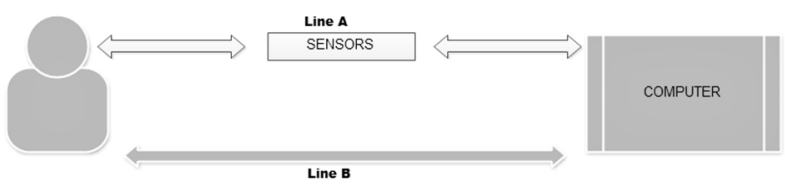

Figure 7. i-Treasures system: sensors' mediation aspects.

In both cases, the use of sensors is the key to reaching the intended results: from the one hand, sensors, by tracking the experts' performance, enable the computer to acquire the information needed to model correct behaviors and, from the other, sensors' track the students' performance so as to allow the computer to assess their performance, in order to provide feedback and propose ad-hoc exercises.

Obviously, the sensors' mediation highly affects the interaction between the humans/users and the system and this calls for increased attention, both as to the requirements collection and to the establishment of evaluation criteria. 
An insight into the processes that have led to the requirements definition and the establishment of the evaluation criteria is outlined in the following paragraphs.

In both cases, a two-folded approach has been adopted by taking a technological perspective and, by assuming the final users' perspective; thus, requirements and evaluation criteria have been established based on the soundness and appropriateness of the employed technologies and on the maximization of users' satisfaction.

\section{Requirements Elicitation for the i-Treasures System}

The process of requirements definition started with the identification of the potential users of the i-Treasures system and the broad definition of their needs, which have been collected by all the partners by referring to both, field experts and final users' schools and associations.

Apart from people from the general public, who might have broad 'informative needs' (i.e., finding materials of various formats in the system), and researchers, who might have 'enquiry needs' (i.e., finding on the platform raw data and focused analyses enabling further research), the main intended user of the i-Treasures system is the learner (i.e.: a subject who is supposed to continue the peculiar and/or endangered tradition) (Pozzi et al., 2014).

A learner expects the system to provide not only informative materials, yet, more importantly, the possibility to practice a cultural expression and receive support and feedback concerning the 'quality' of her/his performance.

Thus, in order to support the acquisition of practical skills by the user, the system should:

- capture relevant aspects of each ICH (some of the ICHs considered in the project are still unexplored)

- fuse and analyze the derived data through an accurate process of data modelling

- teach the user main contents and aspects of $\mathrm{ICH}$

- analyze user's performance and provide him with appropriate feedback about the 'correctness' of her/his performance. As a matter of fact, feedback represents a key aspect in all learning systems, but in the i-Treasures case, it is vital, being at the very core of the overall educational approach adopted.

From a conceptual viewpoint, the process of functional requirements definition implied taking into account:

- what data should be captured and how,

- how the data modelling should be carried out,

- the kind of learning activities the system should propose to support effective educational interventions

- the main functionalities needed to acquire and assess users' performance (through a 3D module for sensorimotor learning).

From the final users' perspective, the emerging requirements can be roughly grouped into the following categories:

- requirements related to the physical and sensor dimension, regulating the interaction between human-users and sensors and viceversa (e.g., sensors should not affect or hinder performance and vice versa),

- requirements related to the learning dimension, regulating more specifically interactions between the user and the Learning Management System (LMS) (e.g., The educational platform shall adjust lessons/difficulty levels according to students' characteristics and abilities),

- requirements related to the environmental dimension, regulating interactions of the human users with the environment, including physical objects and places where the performance takes place (e.g., The system shall adapt to the place where the performance happens),

- requirements related to the social dimension, including partner-performers in the case of group activities (e.g., The system should detect physical interactions among performers in a group), and

- requirements related to the content domain, regulating interactions of the users with the system, strongly dependent upon the relationship with specific contents of the learning activities (e.g., for a specific content area of dance: The system shall visually highlight the student's mistakes). 
From a technical viewpoint, specific non-functional requirements were also set, among which those related to the expected functions of the web platform for education. In particular, it was felt as mandatory that the platform should offer equal access opportunities to all users, including those with disabilities, providing interaction facilities to any kind of learners and offering different personalized learning paths.

Following this orientation, Ease of use, Multilingual, Personalized and Universal Access were established as pillar-requirements for the system and the platform (Pozzi et al, 2014).

Among the non-functional requirements, specific attention was devoted to both "Accessibility" (i.e., features guaranteeing actual possibility to access and use of the system to a variety of users with different abilities) and "Usability" (i.e., general aspects involving effectiveness, efficiency and satisfaction of use of the system).

As to Accessibility, considered as particularly important was "the degree to which the platform and related tools were actually available/could be used by as many people as possible", including people with functional/sensory disabilities, such as vision or hearing impairments. Requirements in this area were established to be compliant with existing accessibility legislations (Bocconi et al, 2006; Dini et al, 2007).

As to Usability (Holzinger, 2005), attention is devoted to general clarity of the system and, in particular, following Nielsen \& Loranger (2006), to its overall quality in terms of:

1. promptness with which users learn to use something,

2. efficiency they attain while making use of it,

3. how easy it is for them to remember how to use it,

4. how error-prone it is, and, finally

5. level of satisfaction that they attain from using it.

Overall, design and development of the i-Treasures web platform take into account the seven basic principles of Universal Design (Center for Universal Design at North Carolina State University) listed in the Table below (Table 1).

The overall definition of requirements called for a complex procedure, extensively described in Pozzi et al. (2014), which was carried out during the initial phase of the project and involved direct contribution of the users, expert performers and researchers in several different fields; the same groups of users and experts were also appointed to contribute to the definition of evaluation criteria.

Following this process, as mentioned above, the identified requirements were grouped into 'categories', according to the four modules of the i-Treasures system i.e., the data capture and analysis module, the data fusion and semantic

\begin{tabular}{|l|}
\hline Equitable use - The design makes the system useful and marketable to people with diverse abilities \\
\hline Flexibility in use - The design accommodates a wide range of individual preferences and abilities \\
\hline $\begin{array}{c}\text { Simple and intuitive - The design is oriented to make the system easy to understand, regardless of the } \\
\text { user's experience, knowledge, language skills, or current concentration level }\end{array}$ \\
\hline $\begin{array}{l}\text { Perceptible information - The design allows the system communicating necessary information effectively to } \\
\text { the user, regardless of ambient conditions or the user's sensory abilities }\end{array}$ \\
Tolerance for error - The design minimizes hazards and the adverse consequences of accidental or \\
unintended actions \\
\hline $\begin{array}{l}\text { Low physical effort - The design entails that the system can be used efficiently and comfortably and with } \\
\text { a minimum of fatigue; this principle was partially extended to include due limitation to cognitive efforts }\end{array}$ \\
Size and space for approach and use - The design of the system foresees that appropriate size and space is \\
provided for approach, reach, manipulation, and use regardless of user's body size, posture, or mobility \\
\hline
\end{tabular}

Table 1. Seven basic principles of Universal Design set by the Center for Universal Design at North Carolina State University. 


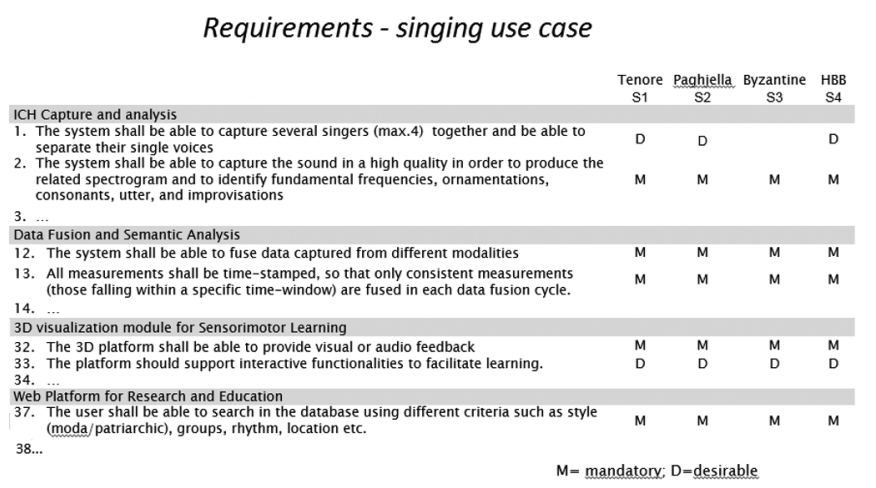

Figure 8. Example of requirements categorization.

analysis module, the 3D module for sensorimotor learning and the web platform for research and education. As an example, Figure 8 shows part of the list of requirements for a singing use case, subdivided into four above mentioned categories.

\section{Assessment Criteria Definition for the i-Treasures System}

The design of the assessment plan was also based on two perspectives, the technical perspective and the user perspective, i.e., performance of the technology used (technical performance) and evaluation of the system's usability (usability evaluation). The technical performance assessment is imperative in order to assure optimization of the developed technological modalities, processing algorithms and interfaces. In this direction, assessment indices, organized in corresponding general categories, are introduced. Following the paradigm of the requirements explained before, these performance indices are divided into functional and non-functional. The functional assessment indices are based on user requirements and systems specifications (as described in subsection 3.1 ), they are module specific and describe the functionality of the module. The non-functional criteria are common across the integrated platform modules and use cases, and they relate to principal properties and characteristics of the system. The usability evaluation is also imperative, since the i-Treasures integrated platform aims towards knowledge exchange. The usability is going to be evaluated through case studies formulated for each sub-use case.
The assessment criteria and indices that will be used for the technical assessment of the integrated platform and its individual modules are divided into two main categories. The first category contains indices that are based on nonfunctional requirements (see previous subsection) and are common across the integrated platform, regardless of the use cases. The second category contains indices that are determined for each module separately, as well as for the data fusion and semantic analysis, Web platform, learning management system and 3D visualization entities. The technical assessment design is partly based on the principles specified in what is also known as the 829 Standard for Software Test Documentation (IEEE 8291998) and adapted to the i-Treasures integrated platform specifications and characteristics.

The non-functional criteria are of significant importance to the usability, sustainability and maintainability of final release of the i-Treasures integrated platform. Criteria reported here conform to the standard for the quality of systems and software (ISO/IEC 25010) and will be taken into consideration during both the two foreseen development cycles of the i-Treasures platform, as well as during the overall assessment of the final system. The general assessment categories are presented in the following Table (Table 2), along with corresponding lists of desired qualities - in the form of questions that will be evaluated.

The main technical assessment indices, organized in more general categories for each module of the i-Treasures separately, are tabulated in Table 3 that follows. 


\begin{tabular}{|c|}
\hline Assessment categories and desired qualities in the form of questions \\
\hline 1. Cost Optimality / Energy efficiency \\
\hline $\begin{array}{l}\text { 1.1) Is the infrastructure (e.g. servers, ups units, broadband services etc.) of the i-Treasures platform } \\
\text { cost- and energy-efficient? }\end{array}$ \\
\hline 1.2) Is the maintenance of the infrastructure cost-efficient? \\
\hline $\begin{array}{l}\text { 1.3) Is it affordable for the single user - institution to acquire the necessary hardware equipment for the } \\
\text { i-Treasures application (e.g. sensors, recording devices)? }\end{array}$ \\
\hline 1.4) Does the user need to install on-fee third party applications for the system to function properly? \\
\hline $\begin{array}{l}\text { 1.5) Does the proper functioning of the i-Treasures platform require updated hardware purchase } \\
\text { (e.g. personal computer with new processor, amount of RAM etc.)? }\end{array}$ \\
\hline 1.6) Will future versions of i-Treasures require the purchase of additional or upgraded hardware? \\
\hline 2. Accessibility / Usability \\
\hline 2.1) Is the content of the i-Treasures presented in different forms (auditory, text, visual elements)? \\
\hline 2.2) Is the web- platform content rendered properly across different browsers? \\
\hline 2.3) Is the i-Treasures web platform responsive? \\
\hline $\begin{array}{l}\text { 2.4) Are web-pages accessible even when newer web technologies are not available (e.g. JavaScript, } \\
\text { CSS3 style-sheets)? }\end{array}$ \\
\hline 2.5) Does the i-Treasures platform support different languages? \\
\hline 2.6) Does the platform provide clear navigation mechanisms? \\
\hline 2.7) Does the platform support simple and advanced search? \\
\hline 2.8) Is the content presentation consistent across pages? \\
\hline 2.9) Does the platform provide feedback on connected hardware configuration? \\
\hline $\begin{array}{l}\text { 2.10) Are response times for task accomplishment (e.g. application loading, refresh time, search results etc.) } \\
\text { proper and adequate? }\end{array}$ \\
\hline 2.11) Does the i-Treasures web platform comply with the universal access standards? \\
\hline 2.12) Does the i-Treasures web platform allow for personalization? \\
\hline 3. Documentation / Support \\
\hline 3.1) Is there a complete, accurate and clear documentation accompanying the i-Treasures platform? \\
\hline 3.2) Is the documentation partitioned into sections for users, developers and administrators? \\
\hline 3.3) Does the assimilation of the documentation require background or expertise from the users? \\
\hline 3.4) If yes, are there links to supporting information resources? \\
\hline 3.5) Is there a "getting started" or a "how to" guide for different use cases? \\
\hline 3.6) Is the documentation available in the i-Treasures website? \\
\hline 3.7) Is there a web page (e.g. FAQs), a forum or e-mail lists for additional support? \\
\hline 4. Interoperability / Portability \\
\hline 4.1) Are the platform components/modules compatible with third party services? \\
\hline 4.2) Does the platform function on different and commonly available operating systems (OSs)? \\
\hline 4.3) Is the downloadable content compatible with the different OSs? \\
\hline 4.4) Besides personal computers, is the platform compatible with tablets or other mobile devices? \\
\hline 5. Extensibility / Scalability \\
\hline 5.1) Are the core/custom components of the i-Treasures platform modular? \\
\hline 5.2) Is backward compatibility taken into account during upgrades? \\
\hline 5.3) Is the source code well-structured and according to coding standards? \\
\hline 5.4) Can the system be remotely managed? \\
\hline 5.5) Does the servers deployment allow for horizontal scalability? \\
\hline 6. Auditing \\
\hline $\begin{array}{l}\text { 6.1) Does the i-Treasures platform provide reports on user activity (per application) and general } \\
\text { user behavior? }\end{array}$ \\
\hline 6.2) Does the platform support auditing of failed login attempts in order to detect brute for \\
\hline
\end{tabular}


6.3) Is the auditing system centralized and secured?

\section{Security / Privacy}

7.1) Does the i-Treasures platform have an authentication system?

7.2) Does the i-Treasures database support role-based access control based on user privileges?

7.3) Is user data transferred and stored securely (e.g. use of encryption algorithms, HTTPS etc.)?

7.4) Is user data available and to whom?

7.5) Does the system user authentication take measures in cases of misuse (lost or stolen passwords, account locks)?

\section{Fault tolerance / Recoverability}

8.1) Is there a software failure monitoring procedure (e.g. error logs)?

8.2) Are hardware maintenance and software upgrades planned in a systematic way?

8.3) Does the system include regular backups of system components and images?

8.4) Can system backup images be restored on different hardware?

8.5) Does the architecture of the platform allow for autonomous functioning of services when a module fails and is being disabled?

8.6) Is the platform functional in cases when external web dependencies (e.g. Europeana platform) go offline?

\section{Licensing / Copyright}

9.1) Has an appropriate license been adopted?

9.2) Is the type of license clearly stated in all platform aspects (e.g. website, source codes)?

9.3) Do platform components include a copyright statement?

9.4) Is it clearly stated who funded the project, developed the platform and owns the copyright?

9.5) Does the platform have a trademark, that doesn't violate other trademarks?

Table 2. Assessment categories and indices based on non-functional requirements.

\begin{tabular}{|l|}
\hline Assessment categories and indices per category \\
\hline \hline 1. Facial Expression Analysis \\
\hline 1.1) Facial feature tracking accuracy \\
\hline 1.2) Facial Action Unit (AU) recognition accuracy \\
\hline 1.3) Basic emotion recognition accuracy \\
\hline 2. Human Body Motion and Gesture Recognition \\
\hline 2.1) Quality of Human Body Motion and Gesture Recognition structure \\
\hline 2.2) Human Body Motion and Gesture recognition Accuracy \\
\hline 2.3) Sensitiveness of the Human Body Motion and Gesture recognition \\
\hline 2.4) Performance identification accuracy \\
\hline 3. Electroencephalography Analysis \\
\hline 3.1) Quality of EEG recording \\
\hline 3.2) Affective Sate Detection Efficiency \\
\hline 3.3) Computational Time \\
\hline 4. Vocal tract sensing and modelling - Ultrasound analysis \\
\hline 4.1) Quality of US pictures \\
\hline $\begin{array}{l}\text { 4.2) Tongue contour extraction (Validation error; Mean Square of Distance between manual contour used as } \\
\text { ground truth and automatically extracted contour) }\end{array}$ \\
\hline 5. Sound Processing \\
\hline 5.1) Individual drums sounds recognition Accuracy \\
\hline 5.2) Instruments recognition Accuracy \\
\hline 5.3) Fundamental Frequency (F0) Analysis \\
\hline 5.4) Respiration (Inhalation detection F-score; Recognition accuracy for egressive and ingressive sounds) \\
\hline
\end{tabular}




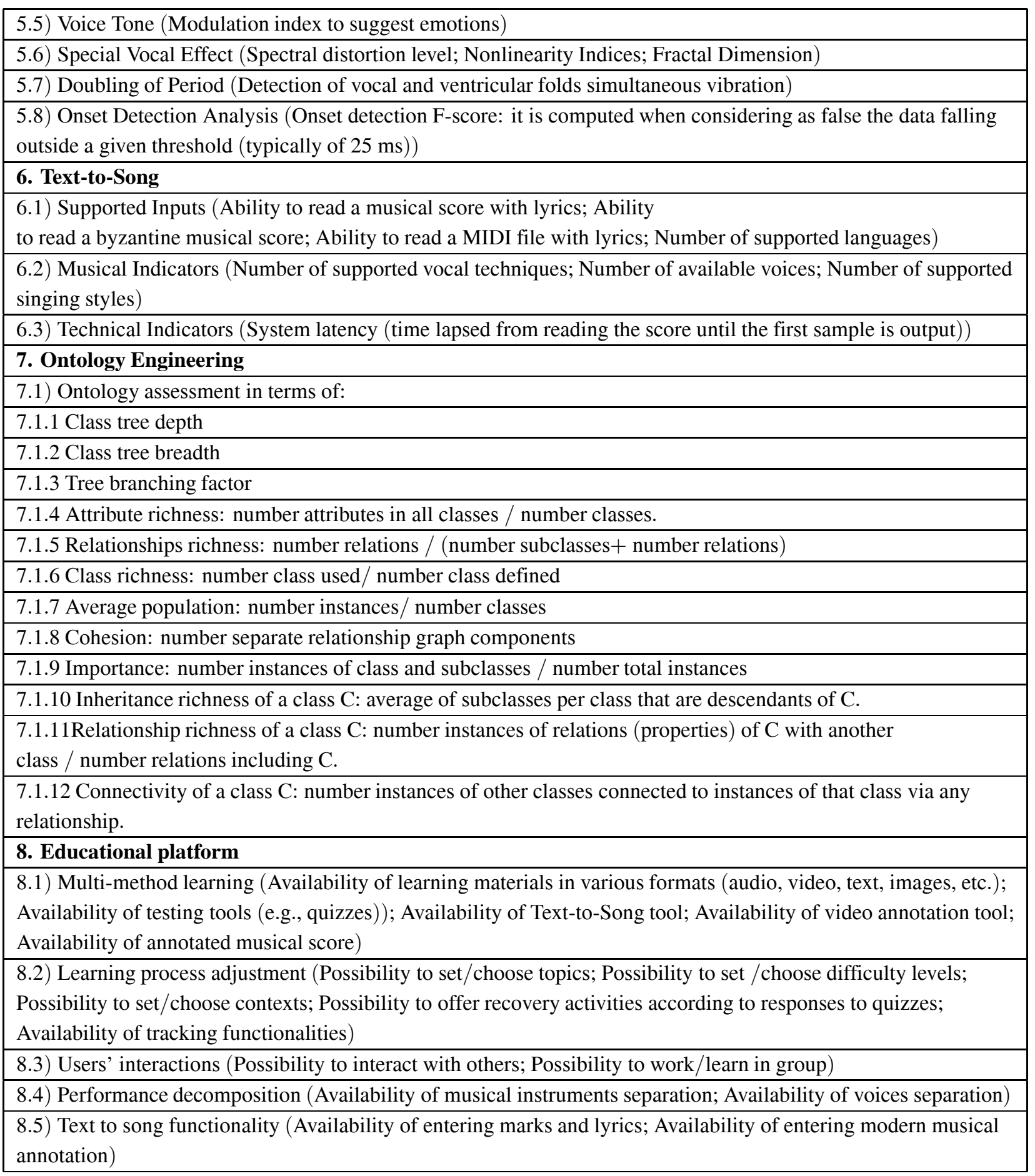

Table 3. Categories of the main technical assessment indices.

\subsection{From Requirements to Evaluation: a Glance to the Iterative Process and its Added Value}

As already mentioned, the overall evaluation of the integrated platform involves both assessment of major technical criteria and indicators and usability evaluation. For the overall evalua- tion to be realized, General Performance Indicators (GPIs) will be generated for each sub-use case and use case. Additionally, the GPI of the integrated platform will be determined that will reflect its overall quality and the degree to which the requirements of the potential users will have been met. The development of the iTreasures platform involves the evaluation of its 
functionalities from the design phase down to the demonstration phase, providing feedback to the development team about any detected shortcomings.

Since the development approach is organized in two cycles, the evaluation for the first cycle (formative evaluation) will constitute the basis for design and development in the second cycle. Figure 9 offers an overview of the expected evolution of the development and assessment/ evaluation processes of i-Treasures. In particular, the assessment of the technical performance (Lab testing) of the first cycle will inform the evaluation of the system's usability ( $1^{\circ}$ case studies). These will provide input for a revision of the original requirements, which will then guide the second development cycle. Similarly to the first cycle, also in the second one, we will have a Lab testing and then the $2^{\circ}$ case studies.

After the completion of the assessment - evaluation process that targets the two development cycles of the i-Treasures platform, the system will be further tested during a final Technical Assessment of the System process (see Figure 9).

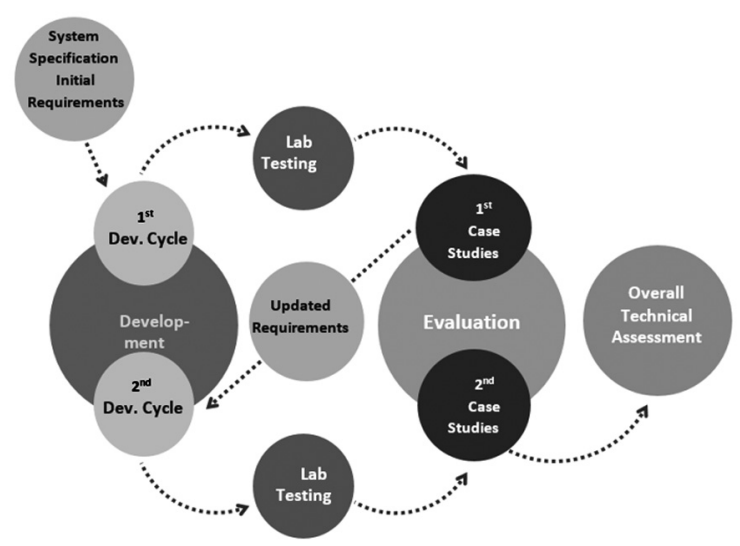

Figure 9. Evolution of the development and assessment - evaluation processes of i-Treasures.

The GPIs will be estimated by fusing technical and usability assessment indices with the aid of fuzzy inference systems (FISs) (Sugeno, 1985). A FIS is a system that uses fuzzy logic sets to map inputs (technical and usability assessment indices) to outputs (more general performance indicators). Here, the Sugeno-type FIS (Sugeno, 1985) will be adopted. A Sugenotype FIS with inputs $x$ and $y$, uses a set of rules to map inputs to the output $z$. A typical rule in a Sugeno fuzzy model is of the type IF-THEN and it has the form

IF input $1=x$ OR Input $2=y$, THEN Output is $z=a x+b y+c$.

The output level $z_{i}$ of each rule is weighted by the firing strength $w_{i}$ of the rule that is derived from the corresponding membership functions $F_{i}($.$) using a logical operation (Sugeno, 1985).$

For each of the modules of the i-Treasures platform, assessment indices will be fused to derive the performance indicator (PI) that characterizes the quality of the module. Thus, five PIs will be produced corresponding to Facial Expression/Modeling, Body and Gesture Recognition, Electroencephalography Analysis, Vocal Tract Sensing/Modeling, Sound Processing and Text-to-Song modules. Moreover, PI of the data fusion and semantic analysis (FSPI) will be estimated separately for each sub-use case of each use case. Furthermore, indices relating to the technical aspects of the LMS, the 3D visualization module and the Web platform will be fused to produce three PIs, respectively.

For the evaluation of the system performance for each sub-use case, appropriate PIs of the ICH capturing and analysis modules, along with the corresponding FSPI will be fused to derive the technical performance indicator (TPI) for each sub-use case. Where applicable, the PI of the $3 \mathrm{D}$-visualization module will be also used. The TPI and the user satisfaction index (USI) arising from the case study relating to the particular sub-use case will then be fuzzified to produce the GPI of the sub-use case. The average of GPIs for all sub-use cases will form the GPI of the use case. Here, there is no need for a FSI as the GPIs of all sub-use cases are of the same significance. The latter process is illustrated in Figure 10.

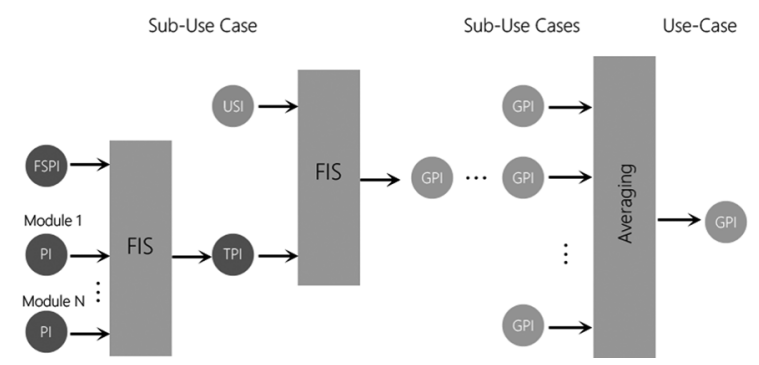

Figure 10. Multilevel fusion of performance indicators (PIs) and user satisfaction index (USI) for the estimation of the general performance indicators (GPIs) of each sub-use case, as well as of the whole use case of the i-Treasures. 
For the evaluation of the quality of the integrated platform, the GPIs of all use-cases along with the PIs of the web platform, the learning management system, and the non-functional requirements fulfillment will be fused to produce the GPI of the overall platform, as illustrated in Figure 11.

Apparently, all the aforementioned indices provide useful feedback in a dynamic way during the development and the functionality of the iTreasures, since they capture both of the two direction phases in the HCI mentioned in Section 3 and could identify both localities as well as more general issues regarding the technical functionality and the user's satisfaction. Moreover, the quantitative and qualitative indices deriving from the assessment categories tabulated in Tables $2 \& 3$ inherently project the degree of flexibility of the system to incorporate a variety of educational uses and adapt to the user. In addition, the fuzzy-logic based approach adopted here further fosters, by definition, the robustness of the assessment and the efficiency in the evaluation processes. In this way, the cycle of the HCI within the i-Treasures is fueled by the continuous monitoring indices, providing effective quantitative means for further development and optimization by its use.

\section{Conclusions}

The paper has briefly discussed the processes of requirements and evaluation criteria definition as they were tackled in the framework of the i-Treasures project. These were regarded as two key aspects of the process, that have led to the building up of the i-Treasures integrated platform having the main goal of widening, as much as possible, the access to ICH (Intangible Cultural Heritage) and fostering its knowledge, learning and, ultimately transmission.

The uniqueness of the i-Treasures endeavor and, in particular, the extensive use of cutting edge sensors for supporting learning and transmission of the rare know-how behind ICH expressions has highly influenced both the requirements elicitation and the establishment of related criteria for the system evaluation. The paper has looked in-depth at these aspects, by outlining some key issues and challenges, which take into account the mediation of sensors in the dialogue between users and the computer.

The process of requirements definition was outlined by showing how it has taken into account the specific characteristics of all the subjects involved (final users' as well as researchers and expert performers) and, in doing so, it has taken both the technical and the users' perspective.

Moreover, a thorough description of the evaluation methodology of the i-Treasures development and functionality was presented, showing the dynamic monitoring of the technical and user's satisfaction issues, via fuzzy logic-based quantitative indices that capture micro- (localities) and macro- (generalities) levels of the iTreasures system use.

In substance, the paper has observed the identification of requirements and the definition of the evaluation criteria, as two different, but closely intertwined processes. In this line, it also emerged that both processes need to be endowed with a high level of plasticity and adaptivity in order to potentially incorporate (and make the most of) possible changes emerging

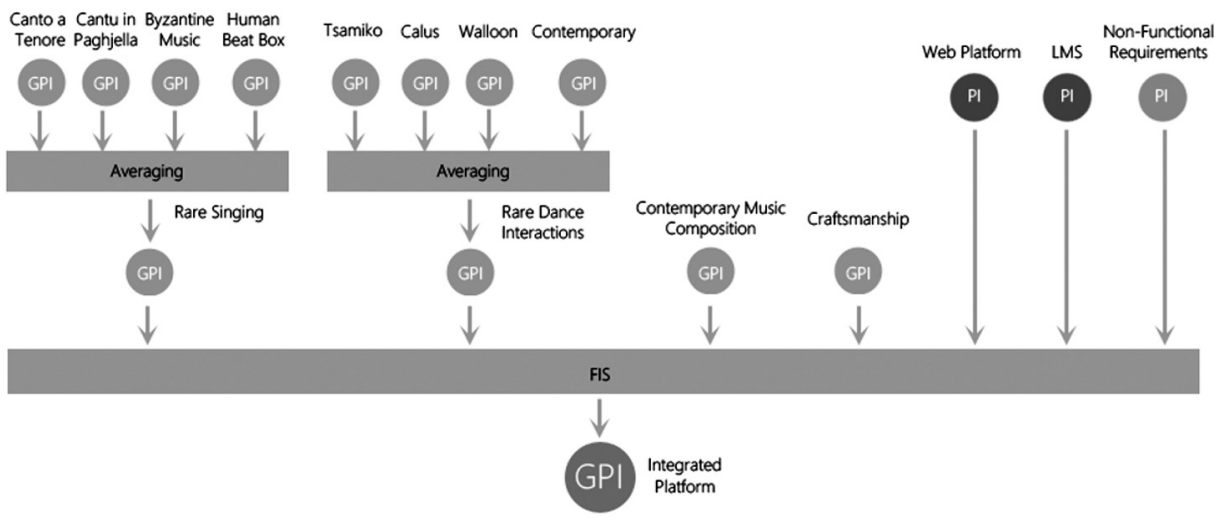

Figure 11. Process of the integrated platform GPI estimation of the i-Treasures. 
in itinere during the phases of system development and testing.

During the following phases of the project, field experiences and demonstrations will be conducted, more aspects will be explored in practice, with the aim to possibly allow revising and updating the existing list of requirements and evaluation criteria, following the iterative and recursive process described.

\section{References}

[1] C. Abras, D. Maloney-Krichmar, J. Preece, User-centered design. In Encyclopedia of HumanComputer Interaction, (W. BAINBRIDGE, ED.), 37(4), pp. 445-56, Thousand Oaks Sage Publications, 2004.

[2] A. Antonaci, M. Ott, F. Pozzi, Virtual Museums, Cultural Heritage Education and $21^{s} t$ Century skills. Proceedings of the ATEE-SIREM Winter Conference, "Learning \& Teaching with Media \& Technology“ (D. PARMigiani, V. Pennazio, A. TRAVERSO, EDS.), Genoa, Italy, 185-195, 2013.

[3] APA GAME http://www.v-must.net/vm-blog /apa-game-short-presentation

[4] K. BECK ET AL., Manifesto for Agile Software Development, Agile Alliance, Available at: http: //agilemanif esto.org. Last retrieved: 30 Jul. 2014 .

[5] S. Bocconi, S. Dini, L. Ferlino, M. OTt, Accessibility of educational multimedia: in search of specific standards. International Journal of Emerging Technologies in Learning (iJET), 3, 1-6, 2006.

[6] S. BøDKER, Scenarios in user-centred designsetting the stage for reflection and action. Interacting With Computers, 13(1), 61-75, 2000.

[7] L. BRANCHESI, The pedagogy of cultural heritage and its evaluation: research areas, methodologies, results and future trends (in Italian). Armando Editore, Roma, 2006.

[8] CENTER FOR UNIVERSAL DESIGN at North Carolina State University http://www. ncsu.edu/ncsu/design/cud/about_ud/udpri nciplestext.htm

[9] A. CockBuRN, Using Both Incremental and Iterative Development. STSC Cross Talk, 21(5), 27-30, 2008.

[10] B. D. Dias, J. A. Diniz, L. J. HadJILEONTIADIS, Towards an intelligent Learning Management System under blended learning. Trends, Profiles and Modelling Perspectives. Springer, Berlin/Heidelberg, 2014.
[11] K. Dimitropoulos, S. Manitsaris et AL. Capturing the Intangible: An Introduction to the ITreasures Project. Proceedings of VISAPP 2014: 9th International Conference on Computer Vision Theory and Applications (S. BATTIATO, J. BRAZ, EDS.), SCITEPRESS - Science and Technology Publications, 2014.

[12] S. Dini, L. Ferlino, A. Gettani, C. Martinoli, \& M. OTT, Educational software and low vision students: evaluating accessibility factors. Universal Access in the Information Society, 6(1), 15-29, 2007.

[13] ETRUSCANNING http://v-must.net/virtu al-museums/vm/etruscanning-3d-2011-2012

[14] A. Gaitatzes, D. Christopoulos, M. Roussou, Reviving the past: cultural heritage meets virtual reality. Proceedings of the 2001 conference on virtual reality, archeology, and cultural heritage, pp. 103-110, ACM, 2001.

[15] GIZA 3D http://giza3d.3ds.com/index. html\#discover

[16] A. HolzINGER, Usability engineering methods for software developers. Communications of the ACM, 48(1), 71-74, 2005.

[17] M. Huo, J. Verner, L. Zhu, \& M. A. BABAr, Software quality and agile methods. Proceedings of the 28th Annual International Conference on Computer Software and Applications. COMPSAC, pp. 520-525. IEEE, 2004.

[18] IEEE 829-1998 Standard For Software Test Documentation http://standards.ieee.org/ findstds/standard/829-1998.html

[19] ISO/IEC 25010 standard for the quality of systems and software http://www. iso.org/iso/home/store/catalogue_ics/ca talogue_detail_ics.htm?csnumber $=35733$

[20] C. LAIRMAN, V. R. BASILI, Iterative and Incremental Development: A Brief History. IEEE Computer, 36(6), pp. 47-56, 2003.

[21] C. LakKa, S. Nikolopoulos, C. VARYTimidis, I. KOMPATSIARIS, A Bayesian network modeling approach for cross media analysis. Signal Processing: Image Communication 26, 175-193, 2011.

[22] LIVIA'S VILLA http://www.vhlab.itabc. cnr.it/flaminia/siti_archeo_villa.html

[23] J. NiELSEN, H. LORANGER, Prioritizing web usability. New Riders Press, Berkeley, CA, 2006.

[24] M. Отт, F. PozzI, ICT and Cultural Heritage Education: Which Added Value? In Emerging Technologies and Information Systems for the Knowledge Society, (M. D. LyTras, J. M. CARROL, E. DAMIANI, R. D. TENNYSON, EDS.), pp. 131-138. Springer, Berlin Heidelberg, 2008.

[25] M. Ott, F. M. Dagnino, F. Pozzi, M. Tavella, Widening Access to Intangible Cultural Heritage: towards the Development of an Innovative platform. Universal Access in Human-Computer Interaction. Universal Access to Information and Knowledge Lecture Notes in Computer Science 8514, 705-713, 2014. 
[26] F. Pozzi, A. Antonaci, F. M. Dagnino, M. Ott, M. TAVELlA, A Participatory Approach to Define User Requirements of a Platform for Intangible Cultural Heritage Education. Proceedings of VISAPP 2014: 9th International Conference on Computer Vision Theory and Applications, (S. BATTIATO, J. BRAZ, EDS.), SCITEPRESS - Science and Technology Publications, 2014.

[27] M. SUGENO, Industrial applications of fuzzy control. Amsterdam, The Netherlands: North-Holland, 1985.

[28] SCROVEGNI CHAPEL http://www.padovan et.it/salamultimediale/index_english.htm

[29] UNESCO, Convention for the Safeguarding of the Intangible Cultural Heritage 2003 Paris, 2003 online at: http://portal.unesco.org/en/ev.php-

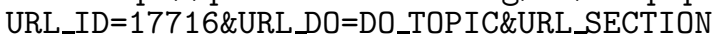
$=201 . \mathrm{html}$

[30] K. H. Veltman, Challenges for ICT/UCT Applications in Cultural Heritage. In CARRERAS, C. (ED.) ICT and Heritage (2005), online at http://www.uoc.edu/digithum/7/dt/eng/do ssier.pdf

Received: August, 2014 Revised: November, 2014 Accepted: November, 2014

Contact addresses: Francesca Dagnino Michela Ot Francesca Pozzi Istituto Tecnologie Didattiche - CNR Italy e-mail: ott@itd.cnr.it

Leontios J. Hadjileontiadis Aristotle University of Thessalonik
FRANCESCA M. DAGNINO is a research fellow at the Istituto per le Tecnologie Didattiche of the Consiglio Nazionale delle Ricerche (CNR). She is a psychologist and a cognitive-behavioural psychotherapist. In the last four years she participated in several international projects. Her main research interests are game based learning, pedagogical planning, and ICT-based solutions for cultural heritage education.

LEONTIOS J. HADJILEONTIADIS (b. 1966) received the Diploma degree in Electrical Engineering in 1989 and the Ph.D. degree in Electrical and Computer Engineering in 1997, both from the Aristotle University of Thessaloniki (AUTH), Thessaloniki, Greece. Dr. Hadjileontiadis also holds a Diploma in Musicology (AUTH, Thessaloniki, 2011) and a Ph.D. degree in Music Composition (University of York, UK, 2004). In December 1999 he joined the Department of Electrical and Computer Engineering, AUTH, Greece as a faculty member, where he is a Professor working on lung sounds, heart sounds, bowel sounds, ECG data compression, affective computing, educational data, seismic data analysis and crack detection in the Signal Processing and Biomedical Technology Unit of the Telecommunications Laboratory. His research interests are in higher-order statistics, alpha-stable distributions, higherorder zero crossings, wavelets, polyspectra, fractals, neuro-fuzzy modeling for medical, mobile and digital signal processing applications.

Michela OTT is a senior researcher at the Istituto per le Tecnologie Didattiche of the Consiglio Nazionale delle Ricerche (CNR), Italy. She presently carries out research in the fields of: cognitive processes underpinning learning, educational use of software tools (including digital games), e-learning effectiveness, learning design, distance education, special education. She has participated in, led and coordinated many national and international research projects in the field of educational technology. She is the author of more than 100 scientific publications published in national and international journals, books and conference proceedings. She is the author of several educational software packages and has contributed to the design and implementation of hypermedia systems.

FRANCESCA POZZI is a researcher at the Istituto per le Tecnologie Didattiche of the Consiglio Nazionale delle Ricerche (CNR), Italy and has got a Ph.D. in Cultures, Languages and ICT (University of Genoa). Pozzi has participated in many national and international projects. Presently, her primary research interests include: computer supported collaborative learning, learning design and ICT-based solutions for cultural heritage education, teacher training, evaluation and learning analytics, MOOCs. 